TITLE:

\title{
Vascular bundle shape in cross- section and relaxation properties of Moso bamboo (Phyllostachys pubescens)
}

\author{
AUTHOR(S): \\ Kanzawa, Eisuke; Aoyagi, Shoko; Nakano, Takato
}

\section{CITATION:}

Kanzawa, Eisuke ... [et al]. Vascular bundle shape in cross-section and relaxation properties of Moso bamboo (Phyllostachys pubescens). Materials Science and Engineering: C 2011, 31(5): 1050-1054

\section{ISSUE DATE:}

2011-07

URL:

http://hdl.handle.net/2433/141952

\section{RIGHT:}

(C) 2011 Elsevier B.V.; This is not the published version. Please cite only the published version.; この論文は出版社版でありません。引用の際に は出版社版をご確認ご利用ください。 
1 Short title: Vascular bundles shape and relaxation properties of bamboo

2

3

4 5

6

7

8

9

10 (1) 21

Vascular bundle shape in cross-section and relaxation properties of Moso bamboo (Phyllostachys pubescens)

Eisuke Kanzawa ${ }^{1}$, Shoko Aoyagi ${ }^{2}$, Takato Nakano, ${ }^{1, *}$

Keywords; Bamboo, Vascular bundle, Image analysis, creep

${ }^{1}$ Laboratory of Biomaterials Design, Division of Forest and Biomaterials Science, Graduate School of Agriculture, Kyoto University.

${ }^{2}$ ASAHI WOODTECH COMPANY, Cyuuou-ku, Osaka, 541-0054, Japan

* Corresponding author; Laboratory of Biomaterials Design, Division of Forest and Biomaterials

Science, Graduate School of Agriculture, Kyoto University, Kita-Shirakawa, Kyoto, 606-8502 Japan. 
22

\section{Abstract}

The variation in the longitudinal and radial direction of the R/T ratio and area ratio of vascular bundles, which are cross-sectional image features of bamboo, and the relationships between the R/T ratio or area ratio and relaxation behavior were investigated. These image features varied characteristically in the longitudinal and radial direction. Relationships between these image features and relaxation behavior was evaluated using the instantaneous creep compliance $\ln [J(0)]$ and the creep intensity $\ln \left[\mathrm{J}\left(3 \times 10^{4}\right)-\mathrm{J}(0)\right]$. Although both instantaneous compliance and creep intensity decreased as density increased, their dependence properties were remarkably different. Instantaneous compliance was strongly correlated with $\mathrm{R} / \mathrm{T}$ ratio and density, which was related to the area ratio, whereas creep intensity was weakly correlated with density in a given range. The results indicate a difference between the two relaxation properties, because creep intensity depends more on microstructures, such as the conformation of molecular chains in the substance, or interactions among cells than on $\mathrm{R} / \mathrm{T}$ ratio and area ratio image features.

Key words: Bamboo; Vascular bundle; Shape factor; Cross-section; Creep 


\section{Introduction}

Bamboo is an important forest biomass resource. However, the use of bamboo is limited to handiwork in most cases, although it is sometimes used as a fill material in various composites. Bamboo has characteristic structures and properties, which have been investigated in various respects [1-7]. In recent years, bamboo has been studied for a variety of applications, including its use as a biomimetic model.

The properties of bamboo originate from the composition and distribution of the vascular bundles and parenchyma tissues, which are the main constituents of the central cylinder of the internodes. The organizational structure of bamboo was reported in a review by Liese et al. [8-10]. Bundle sheaths, consisting of thick-walled fibrous cells with multilayered wall structures, surround the vessels and sieve tubes of the vascular bundles. The bundle sheaths provide the vascular bundles with rigidity by stretching continuously in the longitudinal direction of each internode. In contrast, parenchyma tissues, which consist of thin-walled parenchyma cells, contribute little to the rigidity of bamboo. Thus, the mechanical properties of bamboo depend on variation in the distribution and shape of the cross-sections of the vascular bundles protected by bundle sheaths.

Nakato [11] divided the culm wall into four equal layers and examined one vascular bundle cross-section in each layer. He found no clear relationship between the height from the ground of the internodes and the ratio derived by dividing the maximum radius of a vascular bundle in the radial direction by the maximum radius in the tangential direction and that the ratio decreased from the outer to inner layers. However, to our knowledge, no reports have focused on the relationship between the shape of vascular bundles and relaxation properties.

The mechanical properties of bamboo have been discussed in terms of tissue structures, particularly the cross-sectional distribution of vascular bundles [12-23]. Although Low et al. [24] mentioned the relaxation property, which is time dependent, they did not discuss its relationship to organizational structure. Aoyagi and Nakano [23] investigated position dependence, which affects the relationship between relaxation property and higher-order structures. They reported that Nutting's equation could be applied to the creep property of Moso bamboo. However, the relaxation property has not been discussed in relation to the shape of vascular bundles.

In this study, we analyzed variation in the distribution and shape of vascular bundles as well as radial and longitudinal density of Moso bamboo. In addition, the effects of higher-order structures on the creep property are discussed.

\section{Materials and methods}




\subsection{Materials}

We used samples from a 6-year-old Moso bamboo (Phyllostachys pubescens) collected in Shimane, Japan. The bottommost internode was labeled internode 1, and the uppermost internode at the top of the culm was labeled internode 27. Twenty-four internodes were used for the image analysis. The epidermis and endodermis of specimens were removed. In the central cylinder, which made up the rest of the culm, we designated the section nearest to the epidermis the outer layer and that next to the endodermis the inner layer. Photographs of the cross-sections were used for the image analysis. Samples cut to a given size were used for the creep test.

2.2 Image analysis

Internode cross-sections were observed with an incident-light microscope, and cross-sectional images were captured using a CCD camera. The cross-sectional area used for the image analysis was determined arbitrarily for each internode. The cross-sectional images were printed, and then the outline of each vascular bundle was traced by hand onto paper and scanned into a computer. The cross-sectional image was converted into a binary image by painting the inside of the vascular bundle outlines. Thus, the shape, including the vessel interiors and sieve tubes, was analyzed.

The center of gravity, area, maximum length, maximum width, and angle of direction were collected for all vascular bundles within each image. Maximum length was the maximum radius of the culm. Maximum width was the maximum radius perpendicular to the direction of the maximum length. Angle of direction $(\theta)$ was the angle between the line including the maximum length and horizontal axis of the image $\left(0^{\circ} \leq \theta \leq 180^{\circ}\right)$. For this work, the direction of the horizontal line was the same as the tangential direction of the culm.

Vascular bundle shape was evaluated using the $\mathrm{R} / \mathrm{T}$ ratio, which is the ratio of the radially maximum length $(\mathrm{R})$ to the tangentially maximum width $(\mathrm{T})$ of a vascular bundle. Parts of vascular bundles located at the edges of images were not evaluated. The area ratio of vascular bundles was defined as the ratio of the total area of the vascular bundles divided by the entire area of analysis in a cross-sectional image.

\subsection{Creep test}

Specimens for the creep test were taken from the same culm as that used for the image analysis. A 100-mm-long rectangular specimen was cut from the central region of each internode and was shaped into the dimension: $90 \times 1.5 \times 85(\mathrm{~mm})$ in longitudinal, radial, tangential directions of bamboo, respectively. The specimen was boiled for $30 \mathrm{~min}$ to release internal stress, then gradually dried in air at room temperature for 1 week with further drying at $60^{\circ} \mathrm{C}$ under vacuum for 1 day 
before the creep test. The specimen was wrapped in polyethylene film to keep moisture content constant. There was little weight change before and after the creep test for each specimen.

Creep tests were conducted in cantilever bending at room temperature. The load was within $30 \%$ of the proportional limit of the load deformation diagram.

\section{Results and discussion}

\subsection{Fluctuation in the $R / T$ ratio and area ratio of vascular bundles}

Figure 1a shows a typical cross-section of Moso bamboo used in this work. Vascular bundles, surrounded by parenchyma tissues in the region between the epidermis and endodermis, were distributed characteristically. The distribution of vascular bundles became dense, and their shape was elongated in the radial direction from the inner to outer layer (Fig. 1b). The characteristic change in the vascular bundles depended on the position in the longitudinal direction. Although these characteristics are well known, quantitative analyses of the distribution in the longitudinal and radial direction have not been conducted. Here, cross-sectional image features of vascular bundles were evaluated using the $\mathrm{R} / \mathrm{T}$ ratio.

Figure 2 shows the relationship between the $\mathrm{R} / \mathrm{T}$ ratio and the area ratio obtained by image analysis of the cross-sectional images and the internode height. The R/T ratio depends on the internode number or internode height, and the tendencies of dependence were different in the inner and outer layers. The $\mathrm{R} / \mathrm{T}$ ratio in the bottom section of the culm was lower than other sections in both the inner and outer layers. The R/T ratio in the outer layer was almost constant for internodes 5-20 and tended to increase for internodes 21-27. In contrast, it was almost constant above internode 5 in the inner layer. That is, the R/T ratio was constant regardless of internode height except in the bottom sections. The area ratio increased continuously with internode number in both the inner and outer layers. Also, the area ratio was larger in the outer layer than in the inner layer.

Figure 3 compares the change in $\mathrm{R} / \mathrm{T}$ ratio and area ratio in the radial direction for the bottom, middle, and top sections of the culm. Radial and longitudinal fluctuations were observed for both the $\mathrm{R} / \mathrm{T}$ ratio and the area ratio. Notably, the $\mathrm{R} / \mathrm{T}$ ratio in the inner layer hardly depended on internode height.

The mechanical properties of bamboo depend on density [12]. Because bamboo consists of parenchyma tissues and vascular bundles, we investigated the density dependency of the R/T ratio and area ratio, which are cross-sectional image features of vascular bundles. Figure 4 shows the density dependency of the $\mathrm{R} / \mathrm{T}$ ratio and area ratio.

A correlation was observed between area ratio and density, though it became weaker as the 
area of the cavity varied within the vascular bundles, because the area ratio was calculated based on the area including the vessel cavities and sieve tubes. The correlation coefficient was higher in the low-density region but somewhat lower in the high-density region. In contrast, the $\mathrm{R} / \mathrm{T}$ ratio tended to depend on density differently than did the area ratio. Little scatter in the R/T ratio was observed, and the correlation was weak in the inner layer, although $\mathrm{R} / \mathrm{T}$ ratio was strongly correlated with density in the outer layer.

\subsection{Change in vascular bundle area ratio}

As shown in Figure 1, there was variation in the average size of the vascular bundles across the culm wall in the radial direction. The average size of the vascular bundles in the inner and outer layers was evaluated from the relationship between the density of vascular bundles $N r$ and the area ratio $A r$ (Fig. 5). $N r$ and $A r$ are given as $N r=n / A$ and $A r=\sum_{i=1}^{n} a_{i} / A$, respectively, where $n$ is the number of vascular bundles in the cross-sectional region of which area is $A$, and $a_{i}$ is the area of a vascular bundle. From the two equations given above, the following equation is obtained: $A r=\left(\sum_{i=1}^{n} a_{i} / n\right) N r$. Thus, when the relationship between $A r$ and $N r$ is linear, the slope $\sum_{i=1}^{n} a_{i} / n$ gives the average area of a vascular bundle in the region. The slopes obtained from Figure 5 were 0.0642 and 0.1585 in the outer and inner layer, respectively. The average area of a vascular bundle in the inner layer was 2.5 times larger than that in the outer layer, indicating that small vascular bundles are distributed densely in the outer layer, whereas large vascular bundles are scattered throughout the inner layer.

The image analysis of 24 cross-sectional images revealed that $\mathrm{R} / \mathrm{T}$ ratio and area ratio varied longitudinally and radially, and that the size of the vascular bundle itself varied from the inner to the outer layer in the radial direction.

\subsection{Effects of vascular bundle shape and area ratio on relaxation properties}

Creep tests were conducted on the specimens cut from the inner and outer layers of some internodes. Although there are many reports on the mechanical properties of bamboo, there are few reports on its relaxation properties [12, 23], especially in relation to area ratio. Chuma et al. [12] showed that the results of mechanical testing can be described by the mixture rule of vascular bundles and parenchymal tissues. Aoyagi and Nakano [23] investigated the effects of position in the longitudinal and radial direction on creep behavior and reported that there is a characteristic 
dependency on density and the application of Nutting's equation.

We evaluated the relationship between relaxation properties and the $\mathrm{R} / \mathrm{T}$ ratio or area ratio, which are the instantaneous compliance $\mathrm{J}(0)$ and the creep intensity $\mathrm{J}\left(3 \times 10^{4}\right)-\mathrm{J}(0)$. The former is the creep compliance when the measuring time $t$ is zero and the latter is the difference between the values of creep compliance at $t=3 \times 10^{4}$ and $t=0$.

Figure 6 shows the density dependences of the instantaneous compliance and creep intensity. Both instantaneous compliance and creep intensity were negatively correlated with density and tended to decrease with increasing density. However, their density dependences largely differed.

No difference was observed between $\ln [J(0)]$ versus density in the inner layer and that in the outer layer, although $\ln [\mathrm{J}(0)]$ decreased continuously as density increased. Both relations were represented by the same curve. Logarithmic $\mathrm{J}(0)$ in the outer layer of internode No.26 was ten times larger than that in the inner layer of internode No.2, indicating that instantaneous compliance depended on the volume fraction or the ratio of vascular bundles, considering that density dependence is equal to the volume fraction dependence. Therefore, the relationship between the $\mathrm{R} / \mathrm{T}$ ratio or area ratio and the instantaneous compliance was examined, and the results are shown in Figure 7. Clear correlation was observed between the cross-sectional image features and $\ln [\mathrm{J}(0)]$, except for the $\mathrm{R} / \mathrm{T}$ ratio in the inner layer.

The density dependence of the creep intensity was different from that of the instantaneous compliance as shown in Figure 6. That in the outer layer, where density was greater than 0.51, was obviously different from that in the inner layer, where density was lower. The creep intensity was almost constant in internodes 4-22, which are the middle regions of the bamboo culm. The relaxation behavior in the outer layer of the middle region of the culm was constant regardless of density. The relationship between instantaneous compliance and creep intensity is shown in Figure 8. The clear difference shown in Figure 8 indicates that the density dependence of short-term behavior differs from that of long-term behavior.

Figures 6-8 show that creep intensity, a time-dependent mechanical property, was independent of the density in a particular range, although a strong correlation existed between instantaneous compliance and density. This implies that relaxation behavior was independent of the vascular bundle image features in this range, considering the correlation in Figure 4. Therefore, other factors should be involved. The finding that the ratio and shape have no relationship with relaxation behavior indicates that this relaxation behavior is not directly related to formation of vascular bundles or its changes in cross-section. This is probably because relaxation behavior is attributed to the conformation change and interaction of the molecular chains in the substance. This difference appears in the flat region in Figure 6, where the density is $0.5-0.7$, that is, in the outer layer of internodes 4-22. That may be related to the fact that bamboo grows into a large culm despite its small diameter [23]. 


\section{Conclusions}

The R/T ratio and area ratio of vascular bundles, which are cross-sectional image features of bamboo, had a characteristic distribution in both the longitudinal and radial directions. The $\mathrm{R} / \mathrm{T}$ ratio was larger in the outer layer than the inner layer in the radial direction, and smaller in the bottom section than other sections of the longitudinal direction. In the outer layer, the R/T ratio was almost constant for internodes 5-20, whereas it increased as the internode number became larger for internodes greater than internode 20. It was almost constant in the inner layer of internodes greater than internode 5 . The area ratio was larger in the outer layer than the inner layer. It increased continuously with internode number, and the degree of increase was larger in the outer layer. The average vascular bundle area in the inner layer was 2.5 times as large as that in the outer layer.

Although both instantaneous compliance, $\ln [\mathrm{J}(0)]$, and creep intensity, $\ln \left[\mathrm{J}\left(3 \times 10^{4}\right)-\mathrm{J}(0)\right]$, decreased as density increased, the density dependence of $\ln [\mathrm{J}(0)]$ was largely different from that of $\ln$ $\left[\mathrm{J}\left(3 \times 10^{4}\right)-\mathrm{J}(0)\right]$. Instantaneous compliance was strongly correlated with density, which was related to the $\mathrm{R} / \mathrm{T}$ ratio and area ratio, whereas creep intensity had a poor correlation with density. This result suggests that the difference between instantaneous compliance and creep intensity is attributable to the dependence of creep intensity on microstructure, such as the conformation of molecular chains in the substance, more strongly than the $\mathrm{R} / \mathrm{T}$ ratio and area ratio, which are cross-sectional image features. 
234

235

236

237

238

239

240

241

242

243

244

245

246

247

248

249

250

251

252

253

254

255

256

257

258

259

260

261

262

\section{References}

[1] Y. Suzuki, Bulletin of Tokyo University Forests. 36 (1948a) 136.

[2] Y. Suzuki, Bulletin of Tokyo University Forests. 36 (1948b) 206.

[3] Y. Suzuki, Bulletin of Tokyo University Forests. 38 (1950) 181.

[4] M. Ota, Bulletin of the Kyushu University 19 (1951) 25.

[5] M. Ota, Bulletin of the Kyushu University 22 (1953) 87.

[6] M. Ota, Bulletin of the Kyushu University 24 (1955a) 73.

[7] M. Ota, Bulletin of the Kyushu University 25 (1955b) 121.

[8] N. Parameswaran, W. Liese, Wood Sci. Technol. 10 (1976) 231.

[9] N. Parameswaran, W. Liese, Cellulose Chem. Technol. 14 (1980) 587.

[10] W. Liese, Wood Sci. Technol. 21 (1987) 189.

[11] K. Nakato, Bulletin of Kyoto Prefectural University 11 (1959)105.

[12] S. Chuma, M. Hirohashi, T. Ohgama, Y. Kasahara, Zairyo 39 (1990) 847.

[13] V.S. Godbole, S.C. Lakkad, Mater. Sci. Letters 5 (1986) 303.

[14] S. Amada, Y. Ichikawa, T. Munekata, Y. Nagase, H Shimizu, Compos. Part B 28 (1997) 13.

[15] Y. Inokuchi, M. Fushitani, S. Chuma, M. Ozawa, T. Kubo, K. Sato, Mokuzai Gakkaishi 43 (1997) 391.

[16] T.Y. Lo, H.Z. Cui, H.C. Leung, Mater. Lett. 58 (2004) 2595.

[17] H. Takagi, Y. Ichihara, JSME Inter. J. 47 (2004) 551.

[18] A.K. Ray, S. Mondal, S.K. Das, P. Ramachandrarao, J. Mater. Sci. 40 (2005) 5249.

[19] J.F. Ma, W.Y. Chen, L. Zhao, D.H. Zhao, J. Bio. Engi 5 (2008) 231.

[20] L. Zou, H. Jin, W.Y. Lu, X. Li, Mater. Sci. Engi. C29, (2009) 1375.

[21] T.Y. Lo, H.Z. Cui, P.W.C Tang, H.C. Leung, Construction and Building Mater. 22 (2008) 1532.

[22] Z.P. Sha, C.H. Fang, S.X. Huang, G.L. Tian, Wood Sci. Technol. (2009) DOI 10. 1007/s00226-009-0290-1.

[23] S. Aoyagi, T. Nakano, Zairyo 58 (2009) 57.

[24] L.M. Low, Z.Y. Che, B.A. Latella, J. Mater. Res. 21 (2006) 1969. 


\section{Figure captions}

264

Fig. 1 Typical transverse cross-sectional features of Moso bamboo (a) and the schematic shape change in the vascular bundle from the inner to outer layers (b).

Fig. 2 Change in the vascular bundle R/T ratio and area ratio with internode number.

Fig. 3 Change in the vascular bundle R/T ratio and area ratio with cross-sectional position.

Fig. 4 Relationships among area ratio, $\mathrm{R} / \mathrm{T}$ ratio, and bamboo density.

Fig. 5 Relationship between area ratio and the number of vascular bundles per unit area.

Fig. 6 Dependence of instantaneous creep compliance $J(0)$ and creep intensity $J\left(3 \times 10^{4}\right)-J(0)$ on bamboo density.

Fig. 7 Relationship between area ratio and R/T ratio and the instantaneous creep compliance $\mathrm{J}(0)$.

Fig. 8 Relationship between instantaneous creep compliance and creep intensity $\mathrm{J}\left(3 \times 10^{4}\right)-\mathrm{J}(0)$. 
313

314

315

316

317

318

319

320

321

322

323

324

325

326

327 in the vascular bundle from the inner to outer layers, b. a. Cross-section of moso bamboo

b. Schematic shape change

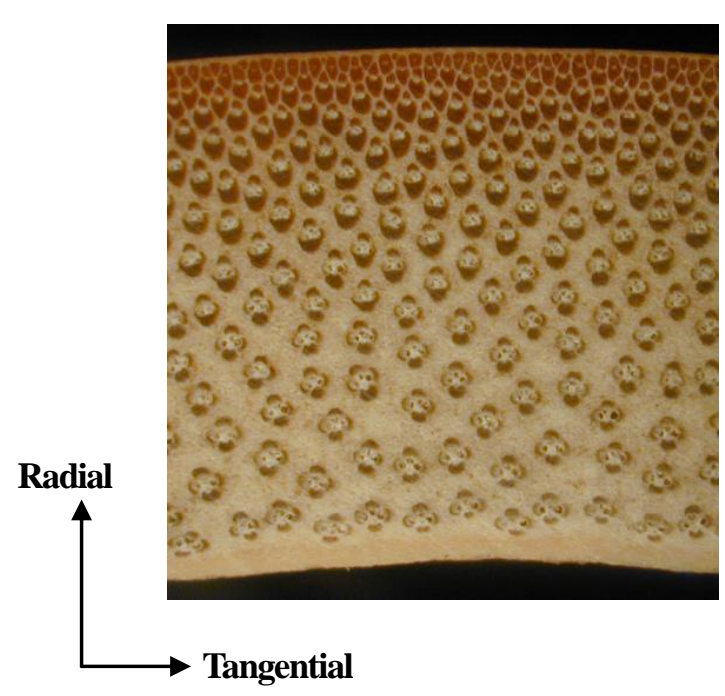

of vascular bundle

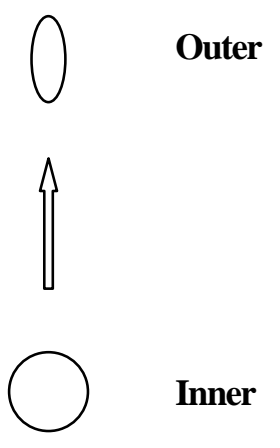

Fig. 1 Typical transverse cross-sectional features of Moso bamboo, a, and the schematic shape change 

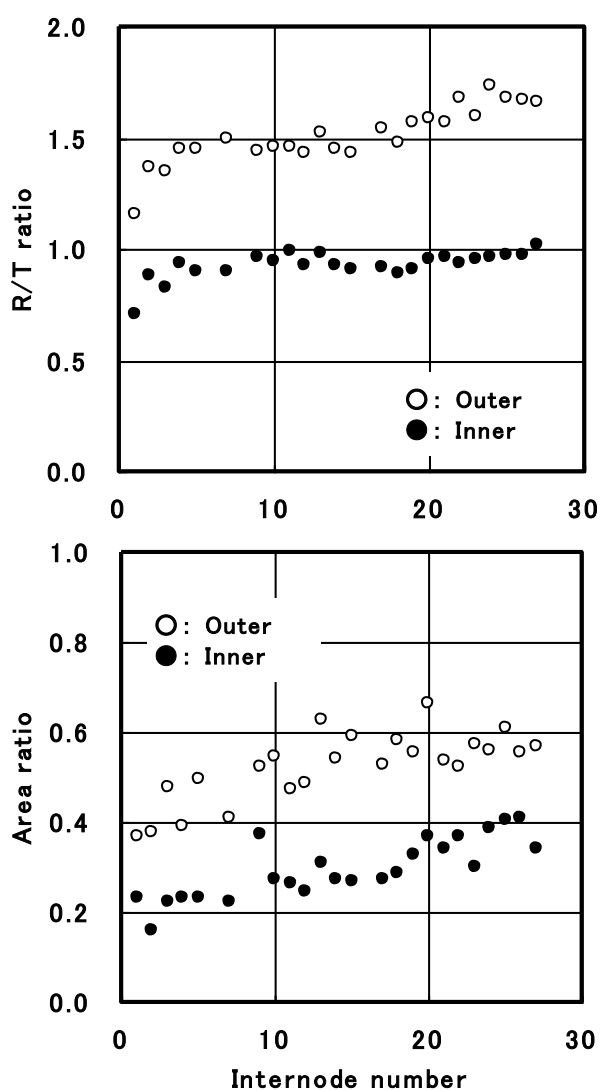

Fig. 2 Change in the vascular bundle $\mathrm{R} / \mathrm{T}$ ratio and area ratio with internode number. 

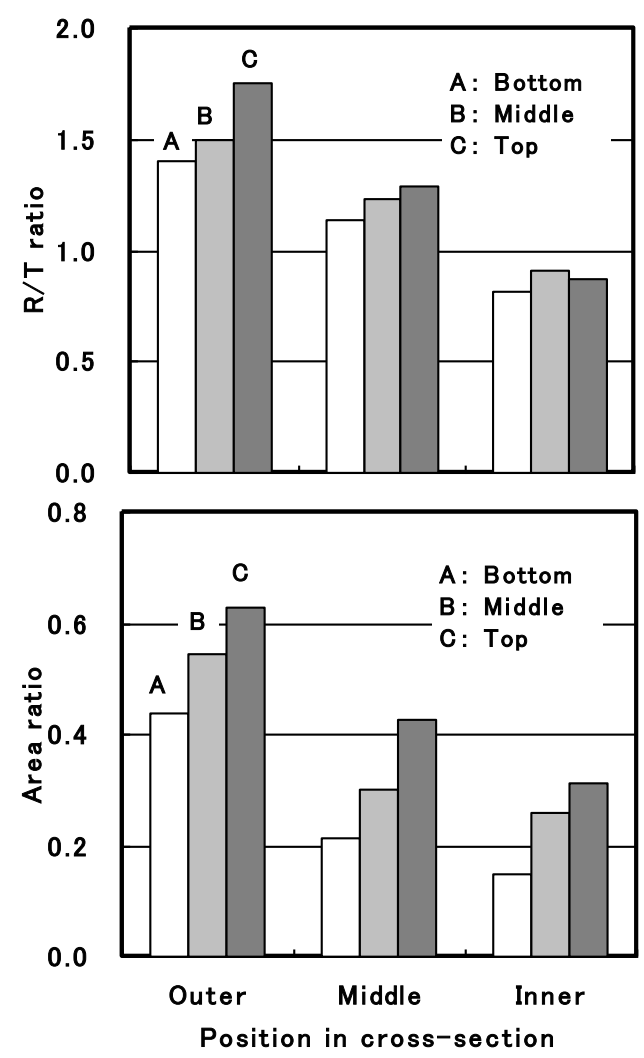

Fig. 3 Change in the vascular bundle $\mathrm{R} / \mathrm{T}$ ratio and area ratio with cross-sectional position. 
379

380

381

382

383

384

385

386

387

388

389

390

391

392

393

394

395

396

397

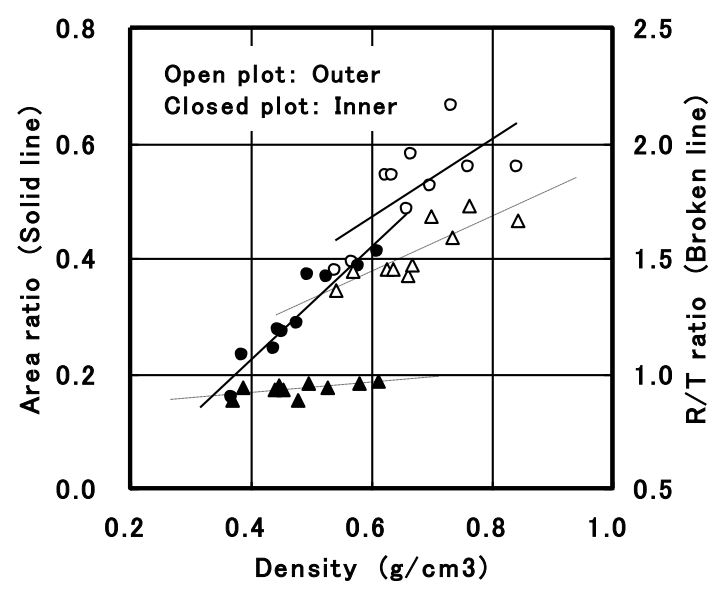

398

399

Fig. 4 Relationships among area ratio, R/T ratio, and bamboo density.

400

401

402

403 
404

405

406

407

408

409

410

411

412

413

414

415

416

417

418

419

420

421

422

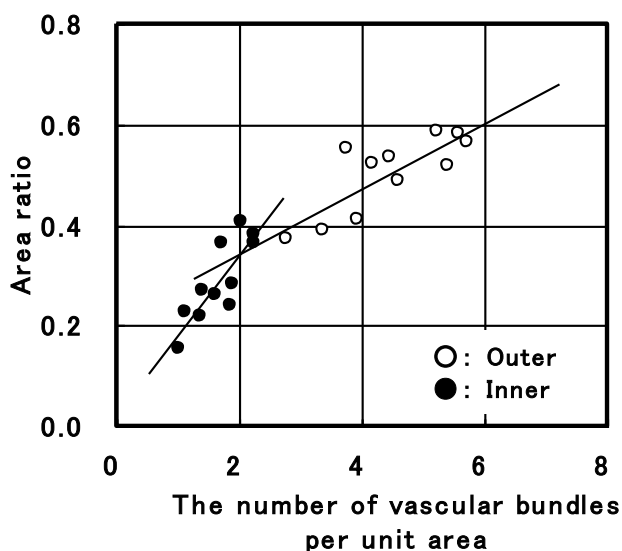

423

424

425

426

Fig. 5 Relationship between area ratio and the number of vascular bundles per unit area.

427

428

429 

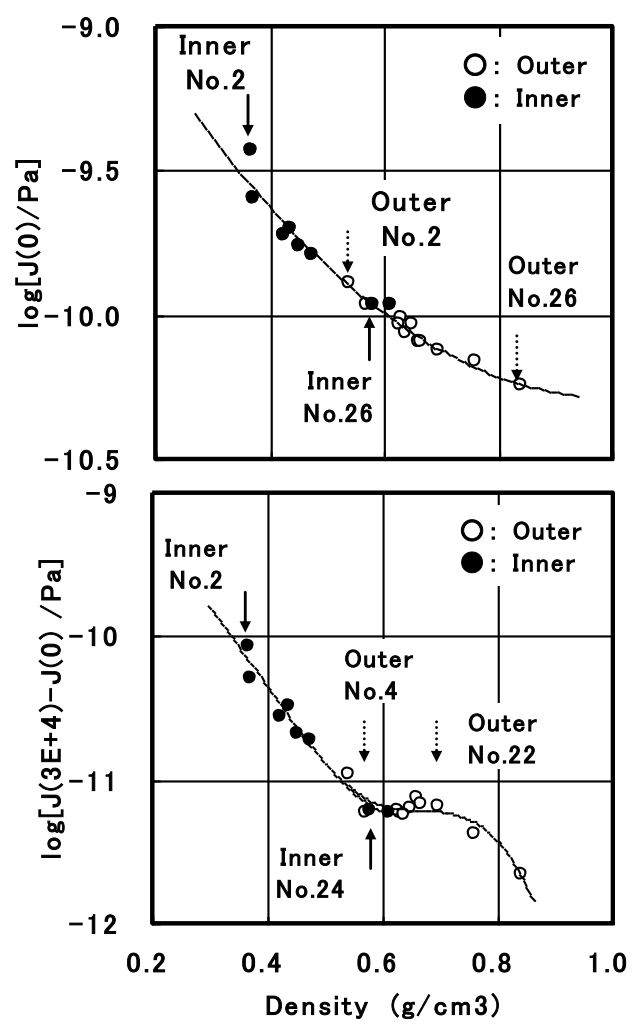

452

Fig. 6 Dependence of instantaneous creep compliance $\mathrm{J}(0)$ and creep intensity $\mathrm{J}\left(3 \times 10^{4}\right)-\mathrm{J}(0)$ on bamboo density. 


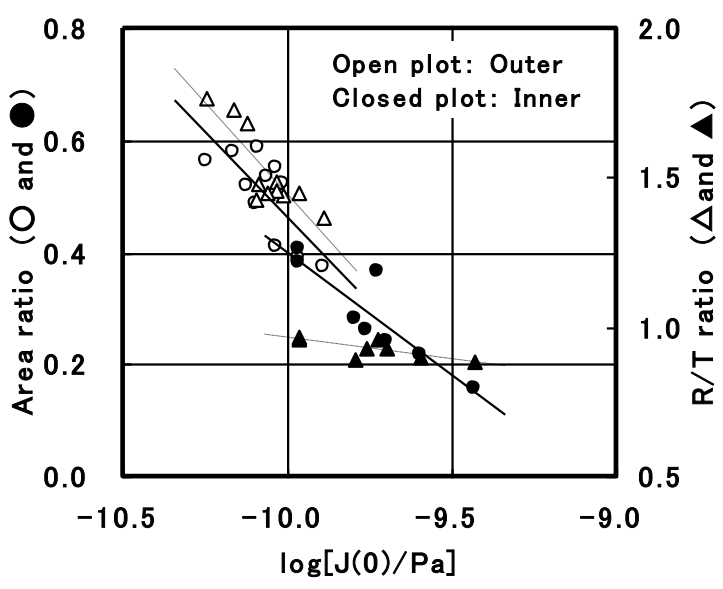

Fig. 7 Relationship between area ratio and R/T ratio and the instantaneous creep compliance $\mathrm{J}(0)$. 


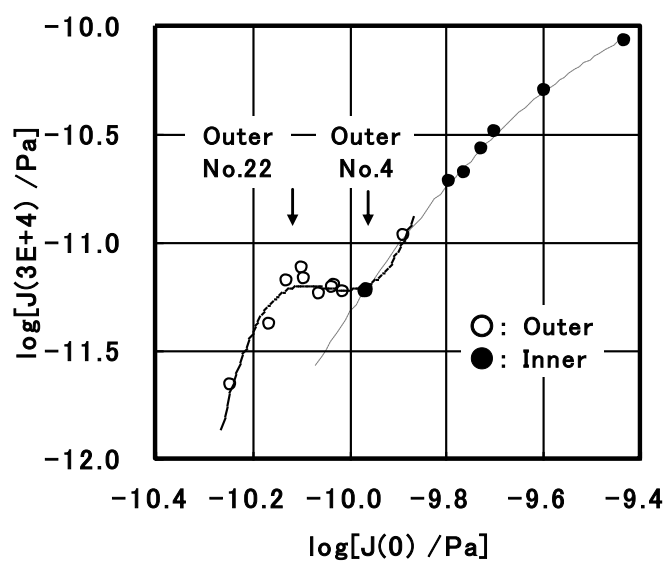

496

497

498

499

Fig.8 Relationship between the instantaneous creep compliance $\mathrm{J}(0)$ and creep intensity $\mathrm{J}\left(3 \times 10^{\wedge} 4\right)-\mathrm{J}(0)$.

500

Fig. 8 Relationship between instantaneous creep compliance and creep intensity $\mathrm{J}\left(3 \times 10^{4}\right)-\mathrm{J}(0)$.

501

502

503

504 